\title{
Baseline Global Longitudinal Strain as a Predictor of Left Ventricular Dysfunction and Hospitalization for Heart Failure of Patients With Malignant Lymphoma After Anthracycline Therapy
}

\author{
Keiko Hatazawa, MD; Hidekazu Tanaka, MD, PhD; Akiko Nonaka, MD, PhD; \\ Hiroki Takada, MD; Fumitaka Soga, MD; Yutaka Hatani, MD; Hiroki Matsuzoe, MD, PhD; \\ Hiroyuki Shimoura, MD, PhD; Junichi Ooka, MD, PhD; Hiroyuki Sano, MD, PhD; \\ Yasuhide Mochizuki, MD, PhD; Kensuke Matsumoto, MD, PhD; Ken-ichi Hirata, MD, PhD
}

\begin{abstract}
Background: Our aim was to investigate the baseline clinical and echocardiographic parameters for predicting left ventricular (LV) dysfunction after anthracycline chemotherapy and heart failure (HF) hospitalization in a single cancer disease.
\end{abstract}

\begin{abstract}
Methods and Results: We studied 73 patients with malignant lymphoma and preserved LV ejection fraction (LVEF). Echocardiography was performed before and after anthracycline chemotherapy. Global longitudinal strain (GLS) was determined from 3 standard apical views. LV dysfunction after anthracycline chemotherapy was defined according to the current definition of cancer therapeuticsrelated cardiac dysfunction. Long-term (50-month) unfavorable outcome was prespecified as hospitalization for HF. A total of 10 patients had LV dysfunction after anthracycline chemotherapy. Multivariate logistic regression analysis showed that baseline GLS was the only independent predictor of this dysfunction. Receiver-operating characteristic curve analysis identified the optimal GLS cutoff for predicting LV dysfunction after anthracycline chemotherapy as $\leq 19 \%(P=0.008)$. Furthermore, the Kaplan-Meier curve indicated that fewer patients with GLS $>19 \%$ were hospitalized for HF than among those with GLS $\leq 19 \%$ (log-rank P=0.02). For sequential logistic models, a model based on baseline clinical variables $\left(X^{2}=2.9\right)$ was improved by the addition of baseline LVEF $\left(X^{2}=9.0 ; P=0.01\right)$, and further improved by the addition of baseline GLS $\left(X^{2}=13.1, P=0.04\right)$.
\end{abstract}

Conclusions: Watchful observation or early therapeutic intervention with established cardioprotective medications may be necessary for patients with malignant lymphoma and preserved LVEF but with abnormal GLS.

Key Words: Echocardiography; Heart failure; Speckle tracking; Ventricular function

$\mathbf{T}$ he mortality rate for patients with various types of cancer has recently decreased because of the diversity of anticancer drugs. However, cancer therapeutics-related cardiac dysfunction (CTRCD) has become a leading cause of morbidity and mortality in survivors, $, 1,2$ and the mortality rate for patients with CTRCD is as high as $60 \%$ by 2 years after treatment, ${ }^{3}$ caused by irreversible left ventricular (LV) myocardial changes due to anticancer drugs, such as myocyte loss, interstitial fibrosis leading to diminished LV contractility, reduced ventricular wall thickness, and progressive LV dilation. Anthracycline is an effective antineoplastic agent used for a wide spectrum of hematologic malignancies and solid tumors, but its most

\section{Editorial p 2477}

serious adverse effect is progressive dose-dependent LV dysfunction followed by congestive heart failure (HF), even years after the treatment has been completed (Type I CTRCD). ${ }^{46}$ Therefore, early detection of LV myocardial damage caused by anthracycline could be important for predicting the possible occurrence of global LV dysfunction or to facilitate early treatment for Type I CTRCD.

Recently, there has been growing interest in early detection of CTRCD by means of global longitudinal strain (GLS) assessed 2D speckle-tracking strain, because it is a

Received March 20, 2018; revised manuscript received May 8, 2018; accepted May 23, 2018; released online July 6, 2018 Time for primary review: 29 days

Division of Cardiovascular Medicine, Department of Internal Medicine, Kobe University Graduate School of Medicine, Kobe (K. Hatazawa, H. Tanaka, H. Takada, F.S., Y.H., H.M., H. Shimoura, J.O., H. Sano, Y.M., K.M., K. Hirata); Division of Cardiology, Hyogo Cancer Center, Akashi (A.N.), Japan

Mailing address: Hidekazu Tanaka, MD, PhD, FACC, FASE, FAHA, FJCS, Division of Cardiovascular Medicine, Department of Internal Medicine, Kobe University Graduate School of Medicine, 7-5-2 Kusunoki-cho, Chuo-ku, Kobe 650-0017, Japan. E-mail: tanakah@med.kobe-u.ac.jp

ISSN-1346-9843 All rights are reserved to the Japanese Circulation Society. For permissions, please e-mail: cj@j-circ.or.jp 
Apical 4-chamber view

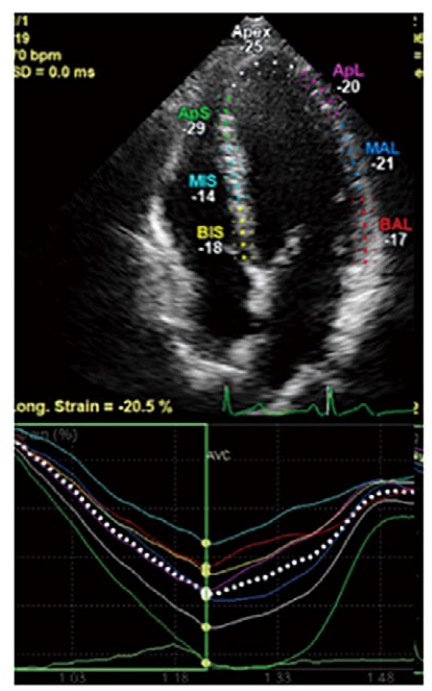

Apical 2-chamberview

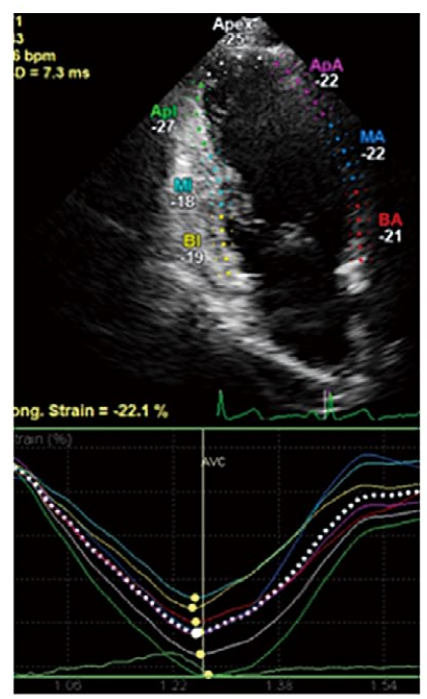

Apical long-axis view

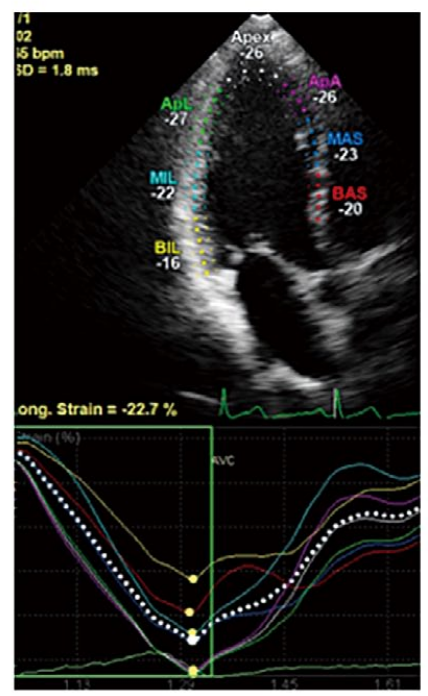

Figure 1. Example of a color-coded 2D display of the left ventricle (LV) and corresponding time-strain curves from 18 LV sites derived from 3 standard apical views for measurement of global longitudinal strain (GLS). GLS was determined as the average peak strain of $18 \mathrm{LV}$ segments, and expressed as an absolute value.

more sensitive and robust parameter for detecting subclinical LV myocardial dysfunction than other conventional LV functional parameters such as LV ejection fraction (LVEF)..$^{7-13}$ In particular, early reduction in GLS during chemotherapy appears to be the most useful parameter for the prediction of CTRCD, although the utility of baseline GLS for predicting CTRCD and the development of HF during long-term follow-up remains unclear. Moreover, previous studies regarding CTRCD seem to be discrepant for reasons such as including different types of cancer for analysis. Our objective was therefore to investigate the baseline clinical and echocardiographic parameters, especially GLS, for predicting cardiotoxicity after undergoing anthracycline chemotherapy for patients with preserved LVEF and a single cancer disease.

\section{Methods}

\section{Study Population}

For this study 78 patients with malignant lymphoma who underwent anthracycline chemotherapy at Hyogo Cancer Center between March 2013 and April 2015 were retrospectively enrolled. Patients were considered eligible if they met the following inclusion criteria: $(1) \geq 18$ years of age; and (2) preserved LV systolic dysfunction, defined as a LVEF $\geq 50 \%$. We excluded patients with: (1) previous history of HF; (2) previous history or suspicion of coronary artery disease, which was carefully assessed by at least 3 senior cardiologists; (3) any known causes of cardiomyopathy; (4) uncontrolled hypertension $>180 / 100 \mathrm{mmHg}$; (5) history of bone marrow transplantation; and (6) more than moderate valvular heart disease. Anemia was defined as a hemoglobin concentration $<12 \mathrm{~g} / \mathrm{dL}$ for women and $<13 \mathrm{~g} / \mathrm{dL}$ for men. We also excluded 5 patients (7\%) from all subsequent analyses because of suboptimal images from poor echocardiographic windows. Accordingly, the patient study group consisted of 73 patients with malignant lymphoma. This study was approved by the local ethics committees of Kobe University Hospital (No. 1807) and Hyogo Cancer Center (R-99).

\section{Echocardiography}

Echocardiographic studies were performed before and after the termination of anthracycline chemotherapy using a commercially available echocardiography system equipped with a 3.5-MHz transducer (iE33; Philips Medical Systems, Andover, MA, USA). Echocardiography after the termination of treatment with anthracycline was performed at the latest within 1 month of completing anthracycline chemotherapy. Digital routine grayscale 2D cine loops from 3 consecutive heart beats were obtained at end-expiratory apnea from standard parasternal and apical views and used for speckle-tracking strain analysis. Sector width was optimized to allow complete myocardial visualization while maximizing the frame rate. Standard echocardiographic measurements were obtained according to the current guidelines of the American Society of Echocardiography/ European Association of Cardiovascular Imaging. ${ }^{14}$ Specifically, LV volumes and LVEF were assessed by modified biplane Simpson's rule using manual tracing of digital images. For patients with atrial fibrillation (AF), measurements of standard echocardiographic and speckle-tracking parameters were acquired as the average of $\geq 3$ consecutive cardiac cycles.

\section{Speckle-Tracking Analysis}

All 2D speckle-tracking strain data were obtained with off-line analysis using dedicated software (QLAB version 10.0 software; Philips Medical Systems). Briefly, the first region of interest was manually traced with the point-and- 
Table 1. Baseline Clinical and Echocardiographic Characteristics of the Patients With Malignant Lymphoma

\begin{tabular}{|c|}
\hline $\begin{array}{c}\text { Patients } \\
(n=73)\end{array}$ \\
\hline $64 \pm 15$ \\
\hline $34 / 39$ \\
\hline $1.6 \pm 0.19$ \\
\hline $117 \pm 14$ \\
\hline $69 \pm 10$ \\
\hline $78 \pm 16$ \\
\hline $10(14)$ \\
\hline $32(44)$ \\
\hline $14(19)$ \\
\hline $41(56)$ \\
\hline $21(29)$ \\
\hline $31(43)$ \\
\hline $11(15)$ \\
\hline $3(4)$ \\
\hline $2(3)$ \\
\hline $2(3)$ \\
\hline $71(97)$ \\
\hline $7(9.6)$ \\
\hline $16(22)$ \\
\hline $15(20.5)$ \\
\hline 35 (47.9) \\
\hline $265 \pm 107$ \\
\hline $8(11)$ \\
\hline $13(18)$ \\
\hline $20(27)$ \\
\hline $29(40)$ \\
\hline $3(4)$ \\
\hline $0(0)$ \\
\hline $18(41)$ \\
\hline $20(27)$ \\
\hline $5(7)$ \\
\hline $35 \pm 6$ \\
\hline $26 \pm 11.9$ \\
\hline $45 \pm 5$ \\
\hline $26 \pm 4$ \\
\hline $9.5 \pm 1.4$ \\
\hline $9.3 \pm 1.4$ \\
\hline $89 \pm 18.5$ \\
\hline $71 \pm 18$ \\
\hline $25 \pm 8$ \\
\hline $65 \pm 5$ \\
\hline $0.9 \pm 0.3$ \\
\hline $7.1 \pm 2.0$ \\
\hline $9.6 \pm 2.8$ \\
\hline
\end{tabular}

\section{Clinical data}

Age, years

Sex (M/F)

Body surface area, $\mathrm{m}^{2}$

Systolic blood pressure, $\mathrm{mmHg}$

Diastolic blood pressure, $\mathrm{mmHg}$

Heart rate, beats/min

Atrial fibrillation, $\mathrm{n}(\%)$

Hypertension, $\mathrm{n}(\%)$

Diabetes mellitus, $\mathrm{n}(\%)$

Either hypertension or diabetes mellitus, $\mathrm{n}(\%)$

Dyslipidemia, n (\%)

Anemia, n (\%)

History of other cancer, $n(\%)$

History of other chemotherapy, $n$ (\%)

History of radiation therapy, $\mathrm{n}(\%)$

Type of malignant lymphoma, $n$ (\%)

Hodgkin lymphoma

Non-Hodgkin lymphoma

Ann Arbor stage, n (\%)

I

II

III

IV

Patients
$(\mathrm{n}=73)$

Cumulative doxorubicin dose, $\mathrm{mg} / \mathrm{m}^{2}$

Distribution of cumulative doxorubicin dose $\left(\mathrm{mg} / \mathrm{m}^{2}\right), \mathrm{n}(\%)$

0-99

100-199

200-299

300-399

400-499

$>500$

(⿻)


Table 2. Comparison of Baseline Clinical and Echocardiographic Characteristics in Patients With and Without LV Dysfunction After Anthracycline Chemotherapy for Malignant Lymphoma

\section{Clinical data}

Age, years

$\operatorname{Sex}(M / F)$

Body surface area, $\mathrm{m}^{2}$

Systolic blood pressure, $\mathrm{mmHg}$

Diastolic blood pressure, $\mathrm{mmHg}$

Patients with LV

dysfunction $(n=10)$

Atrial fibrillation, $\mathrm{n}(\%)$

Hypertension, $\mathrm{n}(\%)$

Diabetes mellitus, $\mathrm{n}(\%)$

Either hypertension or diabetes mellitus, $n(\%)$

Dyslipidemia, n (\%)

Anemia, $\mathrm{n}(\%)$

History of other cancer, $\mathrm{n}(\%)$

History of other chemotherapy, $n$ (\%)

History of radiation therapy, $\mathrm{n}(\%)$

Ann Arbor stage, $n$ (\%)

I

II

III

IV

Cumulative doxorubicin dose, $\mathrm{mg} / \mathrm{m}^{2}$

Distribution of cumulative doxorubicin dose $\left(\mathrm{mg} / \mathrm{m}^{2}\right), \mathrm{n}(\%)$

0-99

100-199

200-299

300-399

400-499

$>500$

\section{Medications}

CCB, $n(\%)$

ACEI/ARB, $n(\%)$

$\beta$-blocker, $\mathrm{n}(\%)$

Echocardiography

LA diameter, $\mathrm{cm}$

$\mathrm{LA}$ volume index, $\mathrm{mL} / \mathrm{m}^{2}$

LV end-diastolic diameter, $\mathrm{mm}$

LV end-systolic diameter, $\mathrm{mm}$

IVST, mm

PWT, mm

LV mass index, $\mathrm{mL} / \mathrm{m}^{2}$

LV end-diastolic volume, $\mathrm{mL}$

LV end-systolic volume, $\mathrm{mL}$

LVEF, \%

$E / A$

$\mathrm{e}^{\prime}, \mathrm{cm} / \mathrm{s}$

$\mathrm{E} / \mathrm{e}^{\prime}$

Speckle-tracking data

GLS, \%

Abbreviations as in Table 1.

click approach on the LV endocardium at the end-systole phase. The second larger region of interest was then generated outside and carefully adjusted near the epicardium. Finally, 6 strain segments and corresponding time-strain curves were generated. The onset point of the QRS com-

\section{Patients without LV dysfunction $(n=63)$ \\ $P$ value}

$61 \pm 16$

$5 / 5$

$1.6 \pm 0.16$

$115 \pm 15$

$67 \pm 7$

3 (30)

4 (40)

$4(40)$

$5(50)$

$4(40)$

$6(60)$

2 (20)

1 (10)

$1(10)$

$1(10)$

0 (0)

$1(10)$

$8(80)$

$279 \pm 114$

0 (0)

3 (30)

2 (20)

$4(40)$

$1(10)$

$0(0)$

1 (10)

2 (20)

$1(10)$

$35 \pm 9$

$32 \pm 15.3$

$46 \pm 6$

$27 \pm 5$

$9.8 \pm 0.9$

$9.6 \pm 1.1$

$96 \pm 19.5$

$85 \pm 19$

$33 \pm 9$

$60 \pm 7$

$0.8 \pm 0.2$

$6.8 \pm 1.4$

$9.9 \pm 2.6$

$18.5 \pm 3.4$

$65 \pm 15 \quad 0.41$

$29 / 34 \quad 0.82$

$1.6 \pm 0.19 \quad 0.94$

$117 \pm 14 \quad 0.69$

$69 \pm 10 \quad 0.60$

$7(11) \quad 0.11$

$28(44) \quad 0.80$

$17(27) \quad 0.41$

$36(57) \quad 0.68$

$17(27) \quad 0.41$

$25(40) \quad 0.23$

$9(35) \quad 0.64$

$2(3) \quad 0.32$

$1(1.5) \quad 0.13$

$6(10) \quad 0.96$

$16(25) \quad 0.07$

$14(22) \quad 0.38$

$27(43) \quad 0.03$

$263 \pm 107 \quad 0.70$

$8(13) \quad 0.24$

$10(16) \quad 0.28$

$18(29) \quad 0.58$

$25(40) \quad 0.99$

2 (3) $\quad 0.32$

$0(0) \quad 1.0$

$17(27) \quad 0.25$

$18(29) \quad 0.58$

$4(6) \quad 0.68$

$35 \pm 6 \quad 0.78$

$25 \pm 11 \quad 0.06$

$45 \pm 5 \quad 0.57$

$26 \pm 4 \quad 0.33$

$9.4 \pm 1.5 \quad 0.43$

$9.2 \pm 1.5 \quad 0.43$

$88 \pm 18.2 \quad 0.19$

$69 \pm 18 \quad 0.01$

$24 \pm 7 \quad<0.001$

$65 \pm 5 \quad<0.01$

$0.9 \pm 0.3 \quad 0.31$

$7.1 \pm 2.1 \quad 0.60$

$9.6 \pm 2.8 \quad 0.73$

$21.6 \pm 2.4$ plex was used as a reference for LV strain analysis. GLS was then determined as the peak strain averaged from the 3 standard apical views as expressed as an absolute value in accordance with current guidelines (Figure 1). ${ }^{14}$ 
Table 3. Univariate and Multivariate Logistic Regression Analyses

\begin{tabular}{|c|c|c|c|c|c|c|}
\hline \multirow{2}{*}{ Variables } & \multicolumn{3}{|c|}{ Univariate analysis } & \multicolumn{3}{|c|}{ Multivariate analysis } \\
\hline & OR & $95 \% \mathrm{Cl}$ & $P$ value & OR & $95 \% \mathrm{Cl}$ & $P$ value \\
\hline \multicolumn{7}{|l|}{ Clinical variables } \\
\hline Age (per 5 years) & 0.983 & $0.943-1.024$ & 0.411 & & & \\
\hline Sex (female) & 1.172 & $0.309-4.455$ & 0.815 & & & \\
\hline Cumulative doxorubicin dose & 1.002 & $0.995-1.001$ & 0.651 & & & \\
\hline Hypertension & 0.833 & $0.214-3.245$ & 0.793 & & & \\
\hline Diabetes mellitus & 1.804 & $0.453-7.185$ & 0.403 & & & \\
\hline Atrial fibrillation & 3.429 & $0.717-16.38$ & 0.123 & & & \\
\hline History of radiation therapy & 6.889 & $0.395-120.1$ & 0.186 & & & \\
\hline \multicolumn{7}{|c|}{ Baseline echocardiographic variables } \\
\hline Left atrial dimension & 1.015 & $0.918-1.122$ & 0.780 & & & \\
\hline LVEF (per 5\%) & 0.832 & $0.723-0.957$ & 0.019 & & & \\
\hline E/e' (per 5 unit) & 1.044 & $0.825-1.321$ & 0.723 & & & \\
\hline GLS & 0.652 & $0.489-0.869$ & 0.004 & 0.652 & $0.489-0.869$ & 0.004 \\
\hline
\end{tabular}

$\mathrm{Cl}$, confidence interval; OR, odds ratio. Other abbreviations as in Table 1.

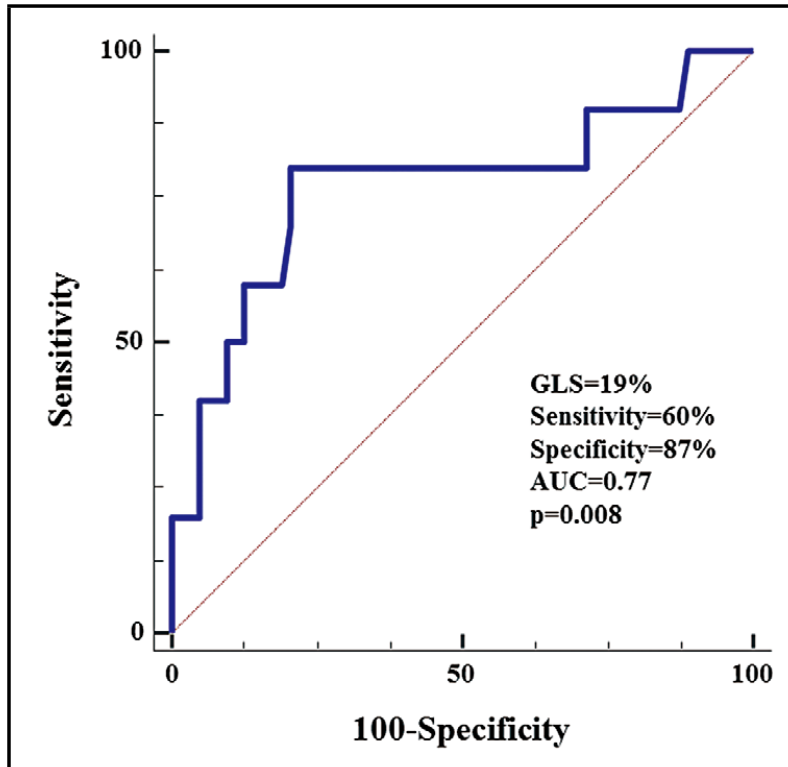

Figure 2. Baseline global longitudinal strain (GLS) $\leq 19 \%$ predicted LV dysfunction after anthracycline chemotherapy. AUC, area under the curve.

\section{Definitions of LV Dysfunction After Anthracycline Chemotherapy and Long-Term Outcome Analysis}

After the cessation of anthracycline chemotherapy, echocardiography was performed for all patients. According to the current definition of CTRCD, LV dysfunction after anthracycline chemotherapy was defined as the presence of (1) an absolute decrease in LVEF $\geq 10 \%$ to a final value $<53 \%$ in asymptomatic patients or (2) an absolute decrease in $\mathrm{LVEF} \geq 5 \%$ to a final value $<53 \%$ in symptomatic patients. ${ }^{12,15}$ Long-term unfavorable outcome events were prespecified as the primary endpoint of hospitalization for deteriorating HF. For long-term follow-up, all 73 patients were tracked for 50 months.

\section{Statistical Analysis}

Continuous variables are expressed as mean values $\pm \mathrm{SD}$ or percentages, and categorical data are summarized as frequencies and percentages. The parameters of the 2 subgroups were compared by unpaired $t$ test, and the paired $t$ test was used for comparison of continuous variables. Proportional differences were evaluated with Fisher's exact test or $X^{2}$ test as appropriate. Optimal cutoff values for the association of baseline GLS with LV dysfunction after anthracycline chemotherapy were determined on the basis of receiver-operator characteristics (ROC) curve analysis. Event-free survival curves were determined with the Kaplan-Meier method and cumulative event rates were compared by log-rank test. The initial univariate logistic regression analysis to identify univariate predictors of $\mathrm{LV}$ dysfunction was followed by a multivariate logistic regression model using stepwise selection, with $\mathrm{P}$ levels for entry set at $<0.1$. Sequential logistic models were constructed to determine any incremental benefits of baseline GLS compared with clinical and conventional echocardiographic variables. A statistically significant increase in the global $\log$-likelihood $X^{2}$ of the model was defined as an increment in predictive value. No multicollinearity was shown among parameters in this study. The intraclass correlation coefficient was then used to determine inter- and intraobserver reproducibilities for speckle-tracking parameters from 20 randomly selected patients using an identical cine-loop for each view. For all steps, $\mathrm{P}<0.05$ was considered statistically significant. All analyses were performed with commercially available software (MedCalc software version 15.11.4; MedCalc Software, Mariakerke, Belgium).

\section{Results}

\section{Baseline Characteristics}

The baseline clinical and echocardiographic characteristics of the 73 patients with malignant lymphoma are summarized in Table 1. Their mean age was $64 \pm 15$ years, 34 were female, LVEF was $65 \pm 5 \%$, and the cumulative anthracycline dose was $265 \pm 107 \mathrm{mg} / \mathrm{m}^{2}$.

\section{Predictors of LV Dysfunction After Anthracycline Chemotherapy}

Of the 73 patients for whom follow-up echocardiographic data were available, $10(14 \%)$ were diagnosed with LV 

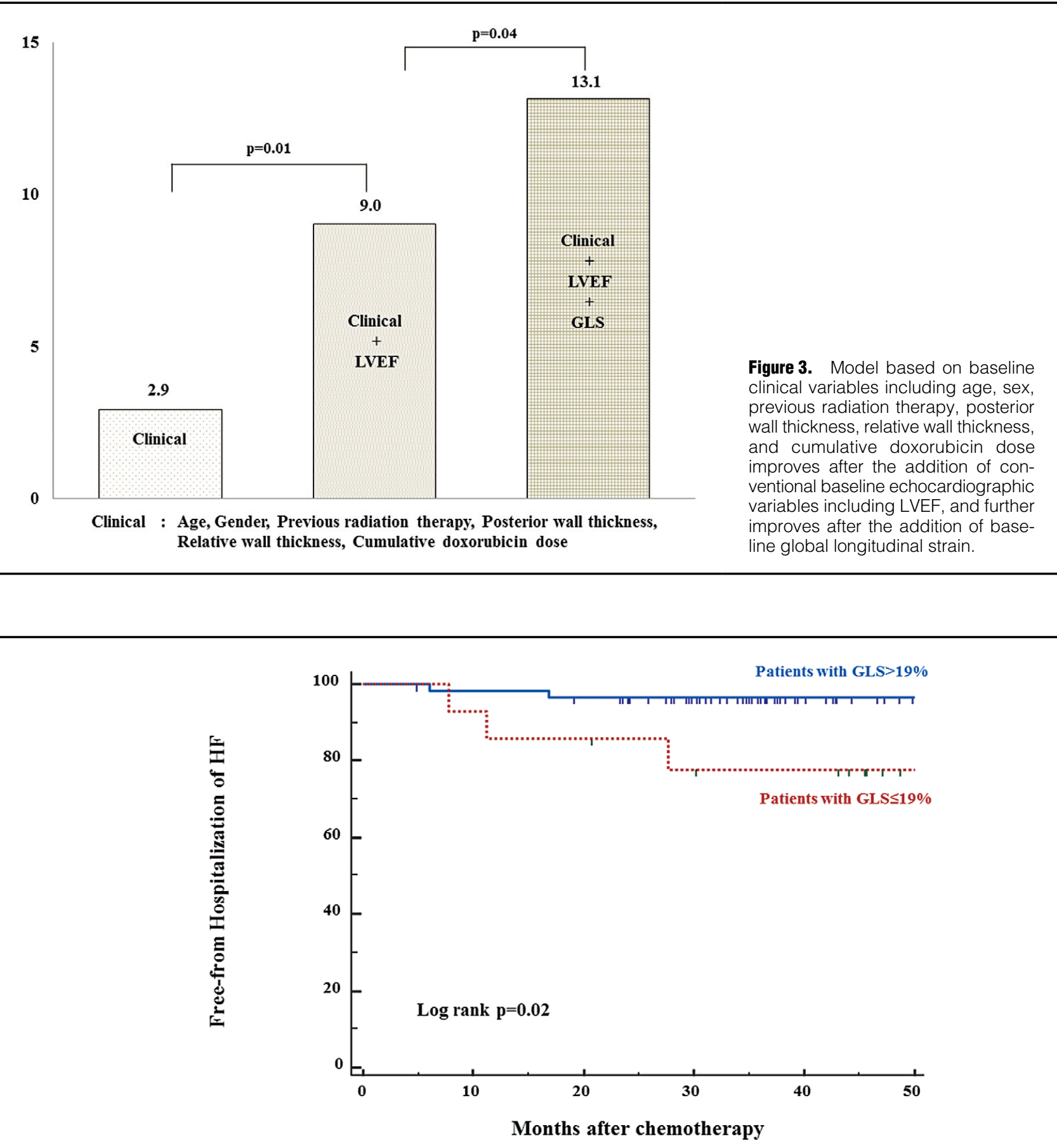

Number at risk

Patients with GLS $>19 \%$

Patients with GLS $\leq 19 \%$
59

14
57

13

55
12

12

42
10

Figure 4. Kaplan-Meier curve shows that patients with GLS $>19 \%$ were hospitalized less frequently for heart failure (HF) than those with GLS $\leq 19 \%$ after anthracycline chemotherapy. GLS, global longitudinal strain.

dysfunction according to the predefined criteria, and the remaining 63 patients $(86 \%)$ were classified as having nonLV dysfunction, all after anthracycline chemotherapy (Table 2). Baseline clinical and echocardiographic parameters were similar except that patients with LV dysfunction after anthracycline chemotherapy were more likely to have a larger LV volume, and lower LVEF and GLS. Moreover, the prevalence of taking angiotensin-converting enzyme inhibitors (ACEI), angiotensin II receptor blockers (ARB), or $\beta$-blockers was similar between groups. Results of uni- 


\begin{tabular}{|c|c|c|c|}
\hline & $\begin{array}{c}\text { Patients with GLS } \\
>19 \%(n=59)\end{array}$ & $\begin{array}{c}\text { Patients with GLS } \\
\leq 19 \%(n=14)\end{array}$ & $P$ value \\
\hline \multicolumn{4}{|l|}{ Clinical data } \\
\hline Age, years & $65 \pm 15$ & $62 \pm 16$ & 0.45 \\
\hline $\operatorname{Sex}(M / F)$ & $29 / 30$ & $5 / 9$ & 0.37 \\
\hline Body surface area, $\mathrm{m}^{2}$ & $1.59 \pm 0.2$ & $1.66 \pm 0.2$ & 0.21 \\
\hline Atrial fibrillation, $\mathrm{n}(\%)$ & $6(10)$ & $4(29)$ & 0.07 \\
\hline Hypertension, $\mathrm{n}(\%)$ & $24(41)$ & $8(57)$ & 0.27 \\
\hline Diabetes mellitus, $\mathrm{n}(\%)$ & $16(27)$ & $5(36)$ & 0.53 \\
\hline Either hypertension or diabetes mellitus, $n(\%)$ & $32(54)$ & $9(64)$ & 0.50 \\
\hline Dyslipidemia, $n(\%)$ & $15(25)$ & $6(43)$ & 0.20 \\
\hline History of another cancer, n (\%) & $7(12)$ & $4(29)$ & 0.12 \\
\hline Previous radiation therapy, $\mathrm{n}(\%)$ & $1(2)$ & $1(7)$ & 0.27 \\
\hline Cumulative doxorubicin dose, $\mathrm{mg} / \mathrm{m}^{2}$ & $266 \pm 103$ & $260 \pm 125$ & 0.85 \\
\hline \multicolumn{4}{|l|}{ Medications } \\
\hline CCB, n (\%) & $14(24)$ & $4(29)$ & 0.71 \\
\hline ACEI/ARB, n (\%) & $17(29)$ & $3(21)$ & 0.58 \\
\hline$\beta$-blocker, n (\%) & $4(6)$ & $1(7)$ & 0.96 \\
\hline Echocardiography & & & - \\
\hline LV end-diastolic diameter, $\mathrm{mm}$ & $45.4 \pm 4.5$ & $44.7 \pm 5.2$ & 0.60 \\
\hline LV end-systolic diameter, mm & $26.0 \pm 4.2$ & $25.7 \pm 4.6$ & 0.79 \\
\hline IVST, mm & $9.3 \pm 1.3$ & $10.1 \pm 1.7$ & 0.08 \\
\hline PWT, mm & $9.1 \pm 1.4$ & $10 \pm 1.4$ & 0.03 \\
\hline Relative wall thickness, mm & $0.41 \pm 0.1$ & $0.46 \pm 0.1$ & 0.03 \\
\hline LV end-diastolic volume, mL & $71 \pm 19$ & $73 \pm 19$ & 0.66 \\
\hline LV end-systolic volume, mL & $24 \pm 7.3$ & $30 \pm 9.8$ & $<0.001$ \\
\hline Left atrial diameter, $\mathrm{mm}$ & $35 \pm 5.7$ & $36 \pm 9.7$ & 0.37 \\
\hline LVEF, \% & $66 \pm 4.5$ & $60 \pm 5.4$ & $<0.001$ \\
\hline LVSV, mL & $46 \pm 12$ & $43 \pm 10$ & 0.33 \\
\hline$E / A$ & $0.9 \pm 0.3$ & $0.9 \pm 0.2$ & 0.59 \\
\hline $\mathrm{e}^{\prime}, \mathrm{cm} / \mathrm{s}$ & $7.1 \pm 2.1$ & $6.8 \pm 1.9$ & 0.55 \\
\hline$E / e^{\prime}$ & $9.3 \pm 2.6$ & $11.0 \pm 3.3$ & 0.04 \\
\hline
\end{tabular}

Abbreviations as in Table 1.

variate and multivariate analyses using logistic regression analysis for LV dysfunction after anthracycline chemotherapy are shown in Table 3. An important finding from the multivariate logistic regression analysis was that GLS was the only independent predictor of LV dysfunction after anthracycline chemotherapy (odds ratio: $0.652 ; 95 \%$ confidence interval $(\mathrm{CI}): 0.489-0.869 ; \mathrm{P}=0.004)$. In addition, ROC curve analysis identified the optimal GLS cutoff for predicting LV dysfunction after anthracycline chemotherapy as $\leq 19 \%$, with a sensitivity of $60 \%$, specificity of $87 \%$, and area under the curve of $0.77(\mathrm{P}=0.008$, Figure 2).

The incremental benefit using sequential logistic models for the prediction of $\mathrm{LV}$ dysfunction after anthracycline chemotherapy is shown in Figure 3. A model based on baseline clinical variables including age, sex, previous radiation therapy, posterior wall thickness, relative wall thickness, and commutative anthracycline dose $\left(\chi^{2}=2.9\right)$ was improved by including conventional baseline echocardiographic variables, including $\operatorname{LVEF}\left(\chi^{2}=9.0 ; \mathrm{P}=0.01\right)$, and further improved by the addition of baseline GLS $\left(\chi^{2}=13.1\right.$, $\mathrm{P}=0.04)$.

\section{Association of GLS With Long-Term Outcome}

The primary endpoint of hospitalization for $\mathrm{HF}$ was recorded for 5 of the 73 patients $(6.8 \%)$. The Kaplan-Meier curve indicated that fewer patients with GLS >19\% were hospitalized for HF than among those with GLS $\leq 19 \%$ after anthracycline chemotherapy (log-rank $\mathrm{P}=0.02$; Figure 4). Some patients were taking ACEI, ARB, or $\beta$-blockers for hypertension or other reasons (Table 1), but none of medications affected the long-term outcomes.

\section{Comparisons of Baseline Parameters of Patients With GLS $>19 \%$ and $\mathrm{GLS} \leq \mathbf{1 9 \%}$}

Because GLS $\leq 19 \%$ was associated with LV dysfunction after anthracycline chemotherapy and hospitalization for HF during long-term follow-up, patients were divided into 2 groups using a cutoff value of GLS $=19 \%$ for a comparison of patient characteristics (Table 4). It was noteworthy that patients with GLS $\leq 19 \%$ were more likely to have LV hypertrophy (LVH: end-diastolic thickness of the interventricular septum (IVS): $10.1 \pm 1.7 \mathrm{~mm}$ vs. $9.3 \pm 1.3 \mathrm{~mm}, \mathrm{P}=0.08$, end-diastolic thickness of the posterior wall: $10 \pm 1.4 \mathrm{~mm}$ vs. $9.1 \pm 1.4 \mathrm{~mm}, \mathrm{P}=0.03$, relative wall thickness: $0.41 \pm 0.1 \mathrm{~mm}$ vs. $0.46 \pm 0.1 \mathrm{~mm}, \mathrm{P}=0.03$ ), and higher early diastolic wave velocity and early diastolic mitral annular velocity ratio (E/e') (11.0 \pm 3.3 vs. $9.3 \pm 2.6, \mathrm{P}=0.04)$ than those with GLS $>19 \%$. Moreover, patients with GLS $\leq 19 \%$ tended to have 
higher prevalence of AF than those with GLS $>19 \%$, but not statistically significant ( $29 \%$ vs. $10 \%, \mathrm{P}=0.07)$.

\section{Reproducibility}

The intraclass correlation coefficient for interobserver reproducibility of GLS was 0.979 (95\% CI: 0.9469-0.9917), and the intraclass correlation coefficient for intraobserver reproducibility of GLS was 0.926 (95\% CI: 0.8122-0.9706).

\section{Discussion}

We found that baseline GLS was the only independent predictor of LV dysfunction after anthracycline chemotherapy for patients with malignant lymphoma and preserved LVEF. In addition, baseline GLS $\leq 19 \%$ was associated with reduced LVEF after anthracycline chemotherapy and hospitalization for HF during long-term follow-up. The lower baseline GLS also yielded significant increments in predictive value compared with conventional clinical echocardiographic variables. This is the first study to demonstrate an association of LV myocardial function before anthracycline chemotherapy with LV systolic dysfunction after anthracycline chemotherapy and hospitalization for HF during long-term follow-up of a single cancer disease.

\section{Association of LV Longitudinal Myocardial Dysfunction With CTRCD}

LVEF is the most common parameter used to assess LV systolic function, and the usefulness of LVEF to detect CTRCD has been previously reported. However, LVEF is an inaccurate parameter of CTRCD because it is insensitive to early changes in cardiac function during a potentially cardiotoxic treatment. Moreover, it is not an accurate predictor of HF of patients who receive anthracycline therapy, because the heart has plenty of reserves and LVEF does not start to deteriorate until the later stages of HF., ${ }^{5,16,17}$ Interest has thus been on the possibility of measuring a more sensitive and robust noninvasive, simple parameter for LV function. In the early stages of $\mathrm{HF}$, or in the case of subclinical LV dysfunction, strain imaging by means of echocardiography can be of considerable help in both diagnostic evaluation and determining prognosis. In this respect, the ability of GLS to predict both subclinical LV dysfunction and cardiovascular outcome may be superior to that of LVEF in a number of cardiac disorders. ${ }^{18,19}$ In fact, some recent investigators have used GLS for the identification of anthracycline-induced early LV longitudinal myocardial dysfunction after chemotherapy. ${ }^{7,8,10,20,21} \mathrm{~A}$ systematic review of 1,504 patients during or after cancer chemotherapy showed that early changes in GLS were the best measure for predicting cardiotoxicity. ${ }^{10}$ Specifically, a $10-15 \%$ early reduction in GLS during chemotherapy appears to be the most useful parameter for predicting cardiotoxicity, defined as a reduction in LVEF or HF. The LV wall is not homogeneous and has 3 layers of fibers, with the endocardial layer often the first to be affected by various diseases. Because this layer is mainly responsible for long-axis contraction, a reduction in longitudinal function has been found to be an early and accurate indicator of LV dysfunction in patients with high susceptibility to CTRCD, as well as ischemia, fibrosis, and hypertro-

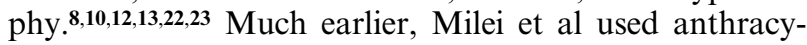
cline-treated rabbits to provide pathologic evidence that anthracycline cardiotoxicity caused progressive vacuoliza- tion of the myocardial fibers, leading to severe myocytolysis in the LV subendocardium and the interventricular septum. ${ }^{23}$ Our group also previously reported that global area strain detected by 3D speckle-tracking imaging, which can quantify the endocardial area change ratio when it is coupled with the factors of both endocardial longitudinal and circumferential strain obtained from all LV segments, was the only parameter independently associated with the cumulative dose in 55 patients with preserved LVEF after undergoing anthracycline chemotherapy. ${ }^{24}$

\section{Clinical Implications}

The association of early LV longitudinal myocardial dysfunction with CTRCD after various types of chemotherapy may have been verified, but the characteristics of LV longitudinal myocardial function before anthracycline chemotherapy in a specific, individual type of cancer disease remain indeterminate. During the long-term followup in our study, we found an association of reduced LVEF after anthracycline therapy and with hospitalization for $\mathrm{HF}$ in patients with malignant lymphoma and baseline GLS $\leq 19 \%$. The lower baseline GLS also yielded significant increments in predictive value compared with conventional clinical echocardiographic variables. The cutoff value of GLS of $19 \%$ used in our study was close to both the normal GLS value of $20 \%$ in the guideline of the American Society of Echocardiography ${ }^{14}$ and the mean normal value of $19.7 \%$ reported in a meta-analysis. ${ }^{25}$ CTRCD may present initially as asymptomatic LV dysfunction and ultimately as symptomatic HF, which can occur even decades after the discontinuation of chemotherapy. Furthermore, Type I CTRCD is believed to be refractory to conventional pharmacological therapy and is associated with a poor prognosis. ${ }^{46}$ Therefore, early detection of Type I CTRCD, preferably before undergoing anthracycline therapy, is crucial, as it will enable early application of preventive strategies with established cardioprotective medications such as ACEI, ARB or $\beta$-blockers for patients with malignant lymphoma and preserved LVEF, but an abnormal baseline GLS. In addition, patients with abnormal baseline GLS are more likely than those with a normal GLS to have $\mathrm{LVH}, \mathrm{AF}$, and higher E/e', which are comorbidities significantly associated with LV longitudinal myocardial dysfunction but preserved LVEF. ${ }^{26}$ Moreover, $\mathrm{LVH}, \mathrm{AF}$, and LV diastolic dysfunction are also considered to be risk factors for the development of CTRCD.,26,27 Thus, watchful observation after anthracycline chemotherapy or after early preventive strategies with established cardioprotective medications but before anthracycline chemotherapy is recommended for patients with malignant lymphoma and preserved LVEF who have such comorbidities.

\section{Study Limitations}

This study had a relatively small number of patients in a retrospective single-center study, so further prospective studies with larger patient populations will be needed to validate our findings. The prevalence of Ann Arbor stage IV in patients with LV dysfunction was significantly higher than in patients without LV dysfunction ( $80 \%$ vs. $43 \%$ ). Although the association of cancer cachexia with the development of LV dysfunction after anthracycline therapy remains uncertain, its effect may be undeniable in this study. Finally, this study enrolled relatively elderly patients (64 \pm 15 years old), but anthracycline is widely used for treatment in various age groups, including young patients. 
Thus, it remains unclear if the cutoff value of GLS $\leq 19 \%$ can be applied to younger patients.

\section{Conclusions}

Baseline GLS was found to be associated with LV dysfunction after anthracycline chemotherapy and the development of HF during long-term follow-up of patients with malignant lymphoma and preserved LVEF. Because anthracycline causes changes in LV performance over time, watchful observation or early therapeutic intervention with established cardioprotective medications may be necessary for such patients with preserved LVEF but abnormal GLS.

\section{References}

1. Hooning MJ, Botma A, Aleman BM, Baaijens MH, Bartelink $\mathrm{H}$, Klijn JG, et al. Long-term risk of cardiovascular disease in 10-year survivors of breast cancer. J Natl Cancer Inst 2007; 99: $365-375$.

2. Doyle JJ, Neugut AI, Jacobson JS, Grann VR, Hershman DL. Chemotherapy and cardiotoxicity in older breast cancer patients: A population-based study. J Clin Oncol 2005; 23: 8597-8605.

3. Felker GM, Thompson RE, Hare JM, Hruban RH, Clemetson DE, Howard DL, et al. Underlying causes and long-term survival in patients with initially unexplained cardiomyopathy. $N$ Engl J Med 2000; 342: 1077-1084.

4. Von Hoff DD, Layard MW, Basa P, Davis HL Jr, Von Hoff AL, Rozencweig M, et al. Risk factors for doxorubicin-induced congestive heart failure. Ann Intern Med 1979; 91: 710-717.

5. Swain SM, Whaley FS, Ewer MS. Congestive heart failure in patients treated with doxorubicin: A retrospective analysis of three trials. Cancer 2003; 97: 2869-2879.

6. Yeh ET, Tong AT, Lenihan DJ, Yusuf SW, Swafford J, Champion $\mathrm{C}$, et al. Cardiovascular complications of cancer therapy: Diagnosis, pathogenesis, and management. Circulation 2004; 109: $3122-3131$.

7. Negishi K, Negishi T, Haluska BA, Hare JL, Plana JC, Marwick $\mathrm{TH}$. Use of speckle strain to assess left ventricular responses to cardiotoxic chemotherapy and cardioprotection. Eur Heart $J$ Cardiovasc Imaging 2014; 15: 324-331.

8. Negishi K, Negishi T, Hare JL, Haluska BA, Plana JC, Marwick $\mathrm{TH}$. Independent and incremental value of deformation indices for prediction of trastuzumab-induced cardiotoxicity. J Am Soc Echocardiogr 2013; 26: 493-498.

9. Negishi T, Negishi K. Echocardiographic evaluation of cardiac function after cancer chemotherapy. J Echocardiogr 2018; 16: $20-27$.

10. Thavendiranathan P, Poulin F, Lim KD, Plana JC, Woo A, Marwick TH. Use of myocardial strain imaging by echocardiography for the early detection of cardiotoxicity in patients during and after cancer chemotherapy: A systematic review. J Am Coll Cardiol 2014; 63: 2751-2768.

11. Plana JC, Galderisi M, Barac A, Ewer MS, Ky B, Scherrer-Crosbie $\mathrm{M}$, et al. Expert consensus for multimodality imaging evaluation of adult patients during and after cancer therapy: A report from the American Society of Echocardiography and the European Association of Cardiovascular Imaging. Eur Heart J Cardiovasc Imaging 2014; 15: $1063-1093$.

12. Plana JC, Galderisi M, Barac A, Ewer MS, Ky B, Scherrer-Crosbie $\mathrm{M}$, et al. Expert consensus for multimodality imaging evaluation of adult patients during and after cancer therapy: A report from the American Society of Echocardiography and the European
Association of Cardiovascular Imaging. J Am Soc Echocardiogr 2014; 27: 911-939.

13. Rhea IB, Uppuluri S, Sawada S, Schneider BP, Feigenbaum H. Incremental prognostic value of echocardiographic strain and its association with mortality in cancer patients. $J$ Am Soc Echocardiogr 2015; 28: 667-673.

14. Lang RM, Badano LP, Mor-Avi V, Afilalo J, Armstrong A, Ernande L, et al. Recommendations for cardiac chamber quantification by echocardiography in adults: An update from the American Society of Echocardiography and the European Association of Cardiovascular Imaging. J Am Soc Echocardiogr 2015; 28: 1-39.e14.

15. Martin M, Esteva FJ, Alba E, Khandheria B, Perez-Isla L, Garcia-Saenz JA, et al. Minimizing cardiotoxicity while optimizing treatment efficacy with trastuzumab: Review and expert recommendations. Oncologist 2009; 14: 1-11.

16. Jensen BV, Skovsgaard T, Nielsen SL. Functional monitoring of anthracycline cardiotoxicity: A prospective, blinded, long-term observational study of outcome in 120 patients. Ann Oncol 2002; 13: $699-709$.

17. Ewer MS, Lenihan DJ. Left ventricular ejection fraction and cardiotoxicity: Is our ear really to the ground? J Clin Oncol 2008; 26: $1201-1203$.

18. Kalam K, Otahal P, Marwick TH. Prognostic implications of global LV dysfunction: A systematic review and meta-analysis of global longitudinal strain and ejection fraction. Heart 2014; 100: $1673-1680$.

19. Gorcsan J 3rd, Tanaka H. Echocardiographic assessment of myocardial strain. J Am Coll Cardiol 2011; 58: 1401-1413.

20. Ho E, Brown A, Barrett P, Morgan RB, King G, Kennedy MJ, et al. Subclinical anthracycline- and trastuzumab-induced cardiotoxicity in the long-term follow-up of asymptomatic breast cancer survivors: A speckle tracking echocardiographic study. Heart 2010; 96: 701-707.

21. Mavinkurve-Groothuis AM, Marcus KA, Pourier M, Loonen J, Feuth T, Hoogerbrugge PM, et al. Myocardial 2D strain echocardiography and cardiac biomarkers in children during and shortly after anthracycline therapy for acute lymphoblastic leukaemia (ALL): A prospective study. Eur Heart J Cardiovasc Imaging 2013; 14: 562-569.

22. Sawaya H, Sebag IA, Plana JC, Januzzi JL, Ky B, Tan TC, et al. Assessment of echocardiography and biomarkers for the extended prediction of cardiotoxicity in patients treated with anthracyclines, taxanes, and trastuzumab. Circ Cardiovasc Imaging 2012; 5: 596-603.

23. Milei J, Boveris A, Llesuy S, Molina HA, Storino R, Ortega D, et al. Amelioration of adriamycin-induced cardiotoxicity in rabbits by prenylamine and vitamins A and E. Am Heart J 1986; 111: $95-102$.

24. Miyoshi T, Tanaka H, Kaneko A, Tatsumi K, Matsumoto K, Minami $\mathrm{H}$, et al. Left ventricular endocardial dysfunction in patients with preserved ejection fraction after receiving anthracycline. Echocardiography 2014; 31: 848-857.

25. Yingchoncharoen T, Agarwal S, Popovic ZB, Marwick TH. Normal ranges of left ventricular strain: A meta-analysis. $J$ Am Soc Echocardiogr 2013; 26: 185-191.

26. Smiseth OA, Torp H, Opdahl A, Haugaa KH, Urheim S. Myocardial strain imaging: How useful is it in clinical decision making? Eur Heart J 2016; 37: 1196-1207.

27. Zamorano JL, Lancellotti P, Rodriguez Munoz D, Aboyans V, Asteggiano R, Galderisi M, et al. 2016 ESC Position Paper on cancer treatments and cardiovascular toxicity developed under the auspices of the ESC Committee for Practice Guidelines: The Task Force for cancer treatments and cardiovascular toxicity of the European Society of Cardiology (ESC). Eur Heart J 2016; 37: $2768-2801$. 